\title{
The average distance and the diameter of dense random regular graphs
}

\author{
Nobutaka Shimizu \\ Department of Mathematical Informatics \\ The University of Tokyo \\ Tokyo, Japan \\ nobutaka_shimizu@mist.i.u-tokyo.ac.jp
}

Submitted: May 1, 2019; Accepted: Sep 3, 2020; Published: Sep 18, 2020

(C) The author. Released under the CC BY-ND license (International 4.0).

\begin{abstract}
Let $\mathrm{AD}\left(G_{n, d}\right)$ be the average distance of $G_{n, d}$, a random $n$-vertex $d$-regular graph. For $d=(\beta+o(1)) n^{\alpha}$ with two arbitrary constants $\alpha \in(0,1)$ and $\beta>0$, we prove that $\left|\operatorname{AD}\left(G_{n, d}\right)-\mu\right|<\epsilon$ holds with high probability for any constant $\epsilon>0$, where $\mu$ is equal to $\alpha^{-1}+\exp \left(-\beta^{1 / \alpha}\right)$ if $\alpha^{-1} \in \mathbb{N}$ and to $\left\lceil\alpha^{-1}\right\rceil$ otherwise. Consequently, we show that the diameter of the $G_{n, d}$ is equal to $\left\lfloor\alpha^{-1}\right\rfloor+1$ with high probability.
\end{abstract}

Mathematics Subject Classifications: 05C80, 05C12

\section{Introduction}

The study of the diameter of regular graphs is well motivated in graph theory. A central question is how to construct an $n$-vertex $d$-regular graph with the minimum possible diameter, which has an application to high-performance computing $[12,17,26]$. Let $D^{\prime}(n, d)$ denote the Moore bound, a well-known lower bound of the minimum possible diameter among all $n$-vertex $d$-regular graphs [26] (we will present the bound in Equation (3)). Let $\operatorname{diam}(G)$ denote the diameter of a graph $G$. We define $\operatorname{diam}(G)=\infty$ if $G$ is not connected. In this paper, we show that the diameter $\operatorname{diam}\left(G_{n, d}\right)$ of a random $d$-regular graph $G_{n, d}$ of $d=(\beta+o(1)) n^{\alpha}$ with two arbitrary constants $\alpha \in(0,1)$ and $\beta>0$ satisfies

$$
\lim _{n \rightarrow \infty}\left(\operatorname{diam}\left(G_{n, d}\right)-D^{\prime}(n, d)\right)= \begin{cases}0 & \text { if either } \alpha^{-1} \notin \mathbb{N} \text { or }\left(\alpha^{-1} \in \mathbb{N} \text { and } \beta<1\right), \\ 1 & \text { if } \alpha^{-1} \in \mathbb{N} \text { and } \beta>1\end{cases}
$$

with probability $1-o(1)$. 
Also, we study the average distance $\operatorname{AD}\left(G_{n, d}\right)$ of a random regular graph. The average distance $\operatorname{AD}(G)$ of a connected graph $G$ is

$$
\operatorname{AD}(G)=\left(\begin{array}{l}
n \\
2
\end{array}\right)^{-1} \sum_{\{u, v\} \in\left(\begin{array}{c}
V \\
2
\end{array}\right)} \operatorname{dist}(u, v)
$$

where $\operatorname{dist}(u, v)$ is the shortest $u v$-path length. If $G$ is not connected, we define $\operatorname{AD}(G)=$ $\infty$.

For a graph property $\mathcal{P}$, we say that an $n$-vertex random graph $G_{n}$ satisfies $\mathcal{P}$ with high probability (w.h.p.) if $\lim _{n \rightarrow \infty} \operatorname{Pr}\left[G_{n}\right.$ satisfies $\left.\mathcal{P}\right]=1$. In this paper, we prove the following results ${ }^{1}$.

Theorem 1. For two constants $\alpha \in(0,1)$ and $\beta>0$, let $d=(\beta+o(1)) n^{\alpha}$ be an integer. For every constant $\epsilon>0$, it holds w.h.p. that

$$
\left|\mathrm{AD}\left(G_{n, d}\right)-\mu\right|<\epsilon
$$

where

$$
\mu= \begin{cases}\alpha^{-1}+\exp \left(-\beta^{1 / \alpha}\right) & \text { if } \alpha^{-1} \in \mathbb{N} \\ \left\lceil\alpha^{-1}\right\rceil & \text { otherwise }\end{cases}
$$

Theorem 2. For two constants $\alpha \in(0,1)$ and $\beta>0$, let $d=(\beta+o(1)) n^{\alpha}$ be an integer. It holds w.h.p. that

$$
\operatorname{diam}\left(G_{n, d}\right)=\left\lfloor\alpha^{-1}\right\rfloor+1 .
$$

The study of $G_{n, d}$ originated from the configuration model introduced by Bollobas [3]. Independently, Bender and Canfield [1] considered a similar model. The configuration model usually enables us to study $G_{n, d}$ for a constant $d$. The case of $d=d(n) \gg 1$ is much less understood, though there is a well-known successful approach called the switching method, introduced by McKay [24]. See [33] for a detailed survey on $G_{n, d}$. However, results shown by the switching method usually require the condition that $d \ll n^{\gamma}$ where $\gamma \leqslant 1$ is some reasonable constant. Therefore, $G_{n, d}$ of $d=(\beta+o(1)) n^{\alpha}$ with arbitrary constant $\alpha$ seems to be far from these methods.

Another recent remarkable approach for the study of $G_{n, d}$ is to compare $G_{n, d}$ with an Erdős-Rényi graph $G(n, p)$ of $p=\frac{d}{n}$. Recall that $G(n, p)$ is an $n$-vertex graph where every two distinct vertices $u$ and $v$ are joined by an edge with probability $p$ independent from any other edges. Since each degree of $G(n, p)$ is concentrated on $n p$, we may expect that $G(n, p)$ and $G_{n, d}$ share several structural properties if $d=(1+o(1)) n p$. For $\log n \ll d \ll$ $n^{1 / 3} /(\log n)^{2}$, Kim and $\mathrm{Vu}[20]$ presented a coupling of $G_{n, d}$ and $G_{n, d}$ of $p=(1-o(1)) \frac{d}{n}$ such that $G(n, p) \subseteq G_{n, d}$ holds w.h.p. Dudek et al. [11, 14] improved this result by presenting a coupling having the same property for $\log n \ll d \ll n$. Their result is

\footnotetext{
${ }^{1}$ In the conference version of this paper [29], we proved Theorem 2.
} 
called the embedding theorem. The embedding theorem enables us to bound $\operatorname{diam}\left(G_{n, d}\right)$ and $\operatorname{AD}\left(G_{n, d}\right)$ from above by $\operatorname{diam}(G(n, p))$ and $\operatorname{AD}(G(n, p))$, respectively. Very recently, Gao, Isaev, and McKay [15] proved that there is a coupling of $G(n, p)$ and $G_{n, d}$ satisfying $G(n, p) \supseteq G_{n, d}$ if $p \geqslant \frac{C d \log n}{n}$ for some constant $C, d=\omega(\log n)$ and $d=o(n)$. We can immediately obtain Theorem 2 by combining the coupling of [15] and known results cencerning the diameter of $G(n, p)$. However, due to the $O(\log n)$ factor in the condition $p \geqslant \frac{C d \log n}{n}$, Theorem 1 does not follow from [15] immediately.

To study $\operatorname{diam}\left(G_{n, d}\right)$ and $\operatorname{AD}\left(G_{n, d}\right)$, we shall look at $\operatorname{diam}(G(n, p))$ and $\operatorname{AD}(G(n, p))$ of $p=\frac{d}{n}$. It is well known that $G(n, p)$ of $p=(\beta+o(1)) n^{-1+\alpha}$ has diameter $\left\lfloor\alpha^{-1}\right\rfloor+1[6,4,14]$. As for the average distance, we obtain a concentration result of $\operatorname{AD}(G(n, p))$, which might be of independent interest.

Theorem 3. For two constants $\alpha \in(0,1)$ and $\beta>0$, let $p=\beta n^{-1+\alpha}$ and

$$
\mu= \begin{cases}\alpha^{-1}+\exp \left(-\beta^{1 / \alpha}\right) & \text { if } \alpha^{-1} \in \mathbb{N}, \\ \left\lceil\alpha^{-1}\right\rceil & \text { otherwise }\end{cases}
$$

Then, there exist absolute constants $C_{1}, C_{2}>0$ such that

$$
|\operatorname{AD}(G(n, p))-\mu| \leqslant C_{1} n^{-C_{2}}
$$

holds w.h.p.

\subsection{Related results and trivial bounds}

Diameter of $\boldsymbol{G}(\boldsymbol{n}, \boldsymbol{p})$. There is a long line of the diameter of $G(n, p)[22,4,8,13,28]$. For dense $G(n, p)$, Bollobas [4] proved the following result.

Theorem 4 (Theorem 6 of [4]). Fix a positive constant c. Let $D=D(n) \geqslant 2$ be a positive integer and $p=p(n) \in[0,1]$ be a real number satisfying

$$
p^{D} n^{D-1}=\log \left(n^{2} / c\right) .
$$

Suppose that $n p=\omega(\log n)$. Then, $G(n, p)$ satisfies

$$
\lim _{n \rightarrow \infty} \operatorname{Pr}[\operatorname{diam}(G(n, p))=k]= \begin{cases}\exp (-c / 2) & \text { if } k=D, \\ 1-\exp (-c / 2) & \text { if } k=D+1 \\ 0 & \text { otherwise. }\end{cases}
$$

Corollary 5. Suppose that $p=(\beta+o(1)) n^{-1+\alpha}$, where $\alpha \in(0,1)$ and $\beta>0$ are any constants. Then, $\operatorname{diam}(G(n, p))=\left\lfloor\alpha^{-1}\right\rfloor+1$ holds w.h.p.

It should be noted that Corollary 5 also follows from the main result of Klee and Larman [22].

The diameter of $G(n, p)$ of small $p$ has gathered special attention $[5,28,8]$. In this line of work, there is a convention that the diameter of a disconnected graph is the 
maximum among all diameters of its connected components. Bollobás [5] proved that $\operatorname{diam}(G(n, p)) \in A$ holds w.h.p. if $n p-\log n=\omega(1)$, where $A=A(n) \subseteq \mathbb{N}$ satisfies $|A| \leqslant 4$. Chung and Lu [8] studied $\operatorname{diam}(G(n, p))$ with $1<n p \leqslant c \log n$ where $c$ is some constant. For example, they proved that $\operatorname{diam}(G(n, p))=(1+o(1)) \frac{\log n}{\log n p}$ holds w.h.p. if $\omega(1)=n p<\log n$. Riordan and Wormald [28] strengthened the results of [8], providing the tight estimate for $\operatorname{diam}(G(n, p))$ for $1+o(1) \leqslant n p=O(1)$. For smaller $p$, Euczak [23] investigated $\operatorname{diam}(G(n, p))$ with $n p<1$.

Average distance of $\boldsymbol{G}(\boldsymbol{n}, \boldsymbol{p})$. The average distance of random graphs with a power law degree sequence has gathered a great deal of attention in network analysis $[18,27,2$, $32,9,31]$. Focusing on $G(n, p)$ with $n p=\omega(\log n)$, one may observe that $\operatorname{AD}(G(n, p)) \approx$ $\operatorname{diam}(G(n, p))$. More precisely, it is easy to see that $\operatorname{AD}(G(n, p)) \leqslant \operatorname{diam}(G(n, p))=$ $(1+o(1)) \frac{\log n}{\log n p}$ and $\operatorname{AD}(G(n, p)) \geqslant(1-o(1)) \frac{\log n}{\log n p}$ hold by considering the maximum degree of $G(n, p))$.

Katzav et al. [18] presented analytical results on $\operatorname{AD}(G(n, p))$ for dense $G(n, p)$ that coincide with Theorem 3. However, to the best of our knowledge, there are no known results with rigorous proofs for $\mathrm{AD}(G(n, p))$ with $n p=n^{\Omega(1)}$.

Diameter of $\boldsymbol{G}_{\boldsymbol{n}, \boldsymbol{d}}$. The diameter of regular graphs has gathered special attention in graph theory $[12,17,26]$ and has an application in designing efficient network topologies. Note that for every vertex $v$, there are at most $d(d-1)^{k}$ vertices having distance $k$ from $v$. Thus, for every $n$-vertex $d$-regular graph $G$ of diameter $D$ with $d \geqslant 3$, we have

$$
\begin{aligned}
D & \geqslant \min \left\{D \in \mathbb{N}: n \leqslant 1+\sum_{i=1}^{D} d(d-1)^{i-1}\right\} \\
& =\left\lceil\log _{d-1} n+\log _{d-1}\left(1-\frac{2}{d}\left(1-\frac{1}{n}\right)\right)\right] \\
& =\frac{\log n}{\log (d-1)}-O(1) .
\end{aligned}
$$

We denote by $D^{\prime}=D^{\prime}(n, d)$ this lower bound Equation (3), which is known as the Moore bound [26].

For random regular graphs $G_{n, d}$, Bollobás and de la Vega [7] proved that

$$
\operatorname{diam}\left(G_{n, d}\right)=D^{\prime}(n, d) \pm O\left(\frac{\log \log n}{\log (d-1)}\right)
$$

holds w.h.p. if the degree $d \geqslant 3$ is a constant. If $\log n \ll d \leqslant n^{o(1)}$, the embedding theorem of Dudek et al. [11, 14] and the lower bound Equation (3) together imply that

$$
\operatorname{diam}\left(G_{n, d}\right)=(1+o(1)) \frac{\log n}{\log d}=(1+o(1)) D^{\prime}(n, d)
$$

holds w.h.p. 
Suppose that $d=(\beta+o(1)) n^{\alpha}$, where $\alpha \in(0,1)$ and $\beta>0$ are constants. From the embedding theorem, we have $\operatorname{diam}\left(G_{n, d}\right) \leqslant\left\lfloor\alpha^{-1}\right\rfloor+1$ holds w.h.p., as we will confirm in Section 2. On the other hand, by substituting $d=(\beta+o(1)) n^{\alpha}$ to Equation (3), we obtain

$$
\lim _{n \rightarrow \infty} D^{\prime}= \begin{cases}\left\lfloor\alpha^{-1}\right\rfloor+1 & \text { if } \alpha^{-1} \notin \mathbb{N} \text { or }\left(\alpha^{-1} \in \mathbb{N} \wedge \beta<1\right), \\ \alpha^{-1} & \text { if } \alpha^{-1} \in \mathbb{N} \wedge \beta>1, \\ \text { depends on the term } o(1) & \text { if } \alpha^{-1} \in \mathbb{N} \wedge \beta=1 .\end{cases}
$$

By combining Theorem 2 and eq. (4), we obtain Equation (1). As mentioned earlier, Theorem 1 immediately follows from the result of Gao, Isaev, and McKay [15]. In this paper, we prove Theorem 2 by combining the upper bound from the embedding theorem [11] and Theorem 1 (note that $\operatorname{diam}(G) \geqslant\lceil\operatorname{AD}(G)\rceil$ ).

Average distance of $\boldsymbol{G}_{\boldsymbol{n}, \boldsymbol{d}}$. Let $N_{k}$ be the number of vertex pairs of distance $k$. We use the same argument as for Equation (3) to obtain a lower bound of $\operatorname{AD}(G)$ for any $d$-regular graph with $d \geqslant 3$. Suppose $\operatorname{diam}(G)=D^{\prime}$ and thus $N_{1}+\cdots+N_{D^{\prime}}=\left(\begin{array}{l}n \\ 2\end{array}\right)$. Moreover, for every $k=1, \ldots, D^{\prime}-1$, we have $N_{k} \leqslant d(d-1)^{k-1}$. Therefore, we obtain

$$
\begin{aligned}
\operatorname{AD}(G) & =\left(\begin{array}{l}
n \\
2
\end{array}\right)^{-1}\left(N_{1}+2 N_{2}+\cdots+D^{\prime} N_{D^{\prime}}\right) \\
& =D^{\prime}-\left(\begin{array}{l}
n \\
2
\end{array}\right) \quad\left(\left(D^{\prime}-1\right) N_{1}+\left(D^{\prime}-2\right) N_{2}+\cdots+N_{D^{\prime}-1}\right) \\
& \geqslant D^{\prime}-\left(\begin{array}{l}
n \\
2
\end{array}\right) \sum_{k=1}^{-1}\left(D^{\prime}-k\right) d(d-1)^{k-1} \\
& =D^{\prime}-\frac{d(d-1)^{D^{\prime}}}{(n-1)(d-2)^{2}}+\frac{d D^{\prime}}{(n-1)(d-2)}+\frac{d}{(n-1)(d-2)^{2}} \\
& =\log _{d-1} n-O(1) .
\end{aligned}
$$

Let $\mathrm{AD}^{\prime}=\operatorname{AD}(n, d)$ denote the lower bound Equation (5). Then, we have

$$
\frac{\log n}{\log (d-1)}-O(1) \leqslant \operatorname{AD}\left(G_{n, d}\right) \leqslant \operatorname{diam}\left(G_{n, d}\right) .
$$

This implies that

$$
\mathrm{AD}\left(G_{n, d}\right)=(1+o(1)) \frac{\log n}{\log (d-1)}
$$

holds w.h.p. if $d \geqslant 3$ is constant or $\log n \ll d \leqslant n^{o(1)}$.

Suppose that $d=(\beta+o(1)) n^{\alpha}$, where $\alpha \in(0,1)$ and $\beta>0$ are constants. From the lower bound Equation (5), we have

$$
\lim _{n \rightarrow \infty} \mathrm{AD}^{\prime}= \begin{cases}\left\lfloor\alpha^{-1}\right\rfloor+1 & \text { if } \alpha^{-1} \notin \mathbb{N}, \\ \alpha^{-1} & \text { if } \alpha^{-1} \in \mathbb{N} \text { and } \beta>1, \\ \alpha^{-1}-\beta^{1 / \alpha}+1 & \text { if } \alpha^{-1} \in \mathbb{N} \text { and } \beta<1, \\ \text { depends on the term } o(1) & \text { otherwise. }\end{cases}
$$




\subsection{Definitions and notation}

For two positive integers $k$ and $m$ with $k \leqslant m$, we denote by $(m)_{k}$ the falling factorial $m(m-1) \cdots(m-k+1)$. For a finite set $X$ and a positive integer $k \leqslant|X|$, we use

$$
\begin{aligned}
& \left(\begin{array}{c}
X \\
k
\end{array}\right):=\left\{\left\{x_{1}, \ldots, x_{k}\right\} \subseteq X:\left|\left\{x_{1}, \ldots, x_{k}\right\}\right|=k\right\}, \\
& (X)_{k}:=\left\{\left(x_{1}, \ldots, x_{k}\right):\left\{x_{1}, \ldots, x_{k}\right\} \in\left(\begin{array}{c}
X \\
k
\end{array}\right)\right\} .
\end{aligned}
$$

For a graph $G$, we denote by $V(G)$ and $E(G)$, respectively, the vertex set and the edge set of $G$. Note that $E(G) \subseteq\left(\begin{array}{c}V(G) \\ 2\end{array}\right)$ is a set of unordered vertex pairs. Throughout the paper, the number of vertices of a graph is denoted by $n$, and the vertex set is denoted by $V=\{1, \ldots, n\}$.

We simply write $H \subseteq G$ if $H$ is contained in $G$, that is, $V(H) \subseteq V(G)$ and $E(H) \subseteq$ $E(G)$ hold. Two graphs $G \cup H$ and $G \cap H$ are defined by

$$
\begin{aligned}
& G \cup H=(V(G) \cup V(H), E(G) \cup E(H)), \\
& G \cap H=(V(G) \cap V(H), E(G) \cap E(H)) .
\end{aligned}
$$

It should be noted that $G$ and $H$ are labelled.

A path is defined to be a graph $P=\left(\left\{v_{0}, \ldots, v_{\ell}\right\},\left\{\left\{v_{0}, v_{1}\right\}, \ldots,\left\{v_{\ell-1}, v_{\ell}\right\}\right\}\right)$ for distinct vertices $v_{0}, \ldots, v_{\ell}$. The vertices of degree one in a path are called endpoints. We call a path of endpoints $s$ and $t$ an st-path. The length of a path is the number of edges. For a graph $G$ and its two distinct vertices $s$ and $t$, the distance $\operatorname{dist}_{G}(s, t)$ is the minimum length among all st-paths contained in $G$. We define $\operatorname{dist}_{G}(s, t)=\infty$ if $G$ does not contain any st-paths. For a graph $G=(V, E)$ of $n$ vertices, the average distance $\operatorname{AD}(G)$ of $G$ is

$$
\mathrm{AD}(G)=\left(\begin{array}{l}
n \\
2
\end{array}\right)^{-1} \sum_{\{s, t\} \in\left(\begin{array}{l}
V \\
2
\end{array}\right)} \operatorname{dist}_{G}(s, t) .
$$

The diameter $\operatorname{diam}(G)$ of $G$ is

$$
\operatorname{diam}(G)=\max _{s \neq t} \operatorname{dist}_{G}(s, t) .
$$

Note that $\operatorname{diam}(G)=\operatorname{AD}(G)=\infty$ if $G$ is not connected. We use $\operatorname{dist}(s, t)$ rather than $\operatorname{dist}_{G}(s, t)$ if the graph $G$ is clear from the context.

For an event $Z$ on a graph $G\left(\right.$ say, $\left.\operatorname{dist}_{G}(1,2) \geqslant \ell\right)$, we use

$$
\mathbb{1}_{[Z]}(G)= \begin{cases}1 & \text { if } G \text { satisfies an event } Z \\ 0 & \text { otherwise }\end{cases}
$$

as the indicator function. 


\subsection{Tools}

Lemma 6 (The Chernoff bound; Theorem 10.1 and 10.5 of [10]). Let $X_{1}, X_{2}, \ldots, X_{n}$ be independent binary random variables satisfying that $\operatorname{Pr}\left[X_{i}=1\right]=p_{i}$ and let $X=\sum_{i=1}^{n} X_{i}$ be the sum. Then, for any $\epsilon \geqslant 0$,

$$
\operatorname{Pr}[X \geqslant(1+\epsilon) \mathbf{E}[X]] \leqslant \exp \left(-\frac{\min \left\{\epsilon, \epsilon^{2}\right\} \mathbf{E}[X]}{3}\right)
$$

and

$$
\operatorname{Pr}[X \leqslant(1-\epsilon) \mathbf{E}[X]] \leqslant \exp \left(-\frac{\epsilon^{2} \mathbf{E}[X]}{2}\right)
$$

Lemma 7 (Multivariate version of Brun's sieve; Lemma 2.8 of [33]). Let $S_{n}^{(1)}, \ldots, S_{n}^{(k)}$ be random variables defined on the same space $\Omega_{n}$ such that each $S_{n}^{(i)}$ can be written as the sum of binary random variables. Suppose that there exist positive constants $\lambda_{1}, \ldots, \lambda_{k}$ satisfying

$$
\lim _{n \rightarrow \infty} \mathbf{E}\left[\prod_{i=1}^{k}\left(S_{n}^{(i)}\right)_{r_{i}}\right]=\prod_{i=1}^{k} \lambda_{i}^{r_{i}}
$$

for every fixed integers $r_{1}, \ldots, r_{k} \geqslant 0$.

Then, for any constants $j_{1}, \ldots, j_{k} \geqslant 0$, it holds that

$$
\lim _{n \rightarrow \infty} \operatorname{Pr}\left[\bigwedge_{i=1}^{k}\left[S_{n}^{(i)}=j_{i}\right]\right]=\prod_{i=1}^{k} \exp \left(-\lambda_{i}\right) \frac{\lambda^{j_{i}}}{j_{i} !} .
$$

Lemma 8 (Lemma 2.1 of [19]). Suppose that $1 \ll d \ll n$. For any fixed graph $H$, it holds that

$$
\operatorname{Pr}\left[H \subseteq G_{n, d}\right]=(1+o(1))\left(\frac{d}{n}\right)^{|E(H)|} .
$$

Let $G[n, m]$ be a graph selected uniformly at random from the set of all graphs of $n$ vertices with exactly $m$ edges.

Lemma 9 (The embedding theorem; Theorem 10.10 of [14]). There is a constant $C>0$ that satisfies the following. For any real $\gamma=\gamma(n)$, integer $d=d(n)$ satisfying

$$
C\left(\left(\frac{d}{n}+\frac{\log n}{d}\right)^{1 / 3}\right) \leqslant \gamma<1
$$

and $m=\lfloor(1-\gamma) n d / 2\rfloor$, there exists a joint distribution $\pi$ of $G[n, m]$ and $G_{n, d}$ such that

$$
\lim _{n \rightarrow \infty} \operatorname{Pr}_{\pi}\left[G[n, m] \subseteq G_{n, d}\right]=1
$$

holds.

In other words, for $\log n \ll d \ll n$, we can choose $m=(1-o(1)) n d / 2$ and couple $G[n, m]$ and $G_{n, d}$ such that $G[n, m] \subseteq G_{n, d}$ holds w.h.p. 


\section{Upper bounds of $\operatorname{AD}\left(G_{n, d}\right)$ and $\operatorname{diam}\left(G_{n, d}\right)$}

In this section we obtain upper bounds of $\operatorname{AD}\left(G_{n, d}\right)$ and $\operatorname{diam}\left(G_{n, d}\right)$ using Lemma 9. As noted in [11], in Lemma 9, one can replace $G[n, m]$ by $G(n, p)$ of $p=(1-2 \gamma) d /(n-1)$. This yields the following result.

Corollary 10. For $d=d(n)$ satisfying $\log n \ll d \ll n$, there exists $p=(1-o(1)) \frac{d}{n}$ such that $\operatorname{AD}\left(G_{n, d}\right) \leqslant \operatorname{AD}(G(n, p))$ and $\operatorname{diam}\left(G_{n, d}\right) \leqslant \operatorname{diam}(G(n, p))$ hold w.h.p.

For $d=(\beta+o(1)) n^{\alpha}$, take $\gamma$ of Lemma 9 satisfying $\gamma=o(1)$, and let $p=(1-2 \gamma) \frac{d}{n-1}=$ $(\beta+o(1)) n^{-1+\alpha}$. Then, from Theorem 3 and corollary 10, it holds w.h.p. that

$$
\mathrm{AD}\left(G_{n, d}\right) \leqslant \operatorname{AD}(G(n, p)) \leqslant \mu+o(1)
$$

Similarly, from Corollaries 5 and 10, a random regular graph $G_{n, d}$ w.h.p. satisfies

$$
\operatorname{diam}\left(G_{n, d}\right) \leqslant \operatorname{diam}(G(n, p)) \leqslant\left\lfloor\alpha^{-1}\right\rfloor+1 .
$$

\section{Lower bounds of $\operatorname{AD}\left(G_{n, d}\right)$ and $\operatorname{diam}\left(G_{n, d}\right)$}

If $\alpha^{-1} \notin \mathbb{N}$, the lower bound Equation (6) and the upper bound Equation (8) yield that

$$
\operatorname{AD}\left(G_{n, d}\right)=\left\lfloor\alpha^{-1}\right\rfloor+1-o(1)
$$

holds w.h.p. Now we focus on the case where $\alpha^{-1} \in \mathbb{N}$. This section is devoted to prove the following.

Lemma 11. Let $d=(\beta+o(1)) n^{\alpha}$, where $\alpha \in(0,1)$ and $\beta>0$ are any constants satisfying $\alpha^{-1} \in \mathbb{N}$. For any constant $\epsilon>0$,

$$
\lim _{n \rightarrow \infty} \operatorname{Pr}\left[\operatorname{AD}\left(G_{n, d}\right) \leqslant \mu-\epsilon\right]=0,
$$

where $\mu=\alpha^{-1}+\exp \left(-\beta^{1 / \alpha}\right)$.

Remark. By combining Equation (8) and lemma 11, we complete the proof of Theorem 1. Moreover, Lemma 11 implies

$$
\operatorname{diam}\left(G_{n, d}\right) \geqslant\left\lceil\operatorname{AD}\left(G_{n, d}\right)\right\rceil=\left\lfloor\alpha^{-1}\right\rfloor+1
$$

holds w.h.p., which completes the proof of Theorem 2.

Proof of Lemma 11. Note that

$$
\operatorname{AD}\left(G_{n, d}\right)=\left(\begin{array}{l}
n \\
2
\end{array}\right)^{-1} \sum_{\{s, t\} \in\left(\begin{array}{c}
V \\
2
\end{array}\right)} \operatorname{dist}(s, t)
$$




$$
\begin{aligned}
& =\sum_{\ell=1}^{\infty}\left(\begin{array}{l}
n \\
2
\end{array}\right)^{-1} \sum_{\{s, t\} \in\left(\begin{array}{c}
V \\
2
\end{array}\right)} \mathbb{1}_{[\operatorname{dist}(s, t) \geqslant \ell]} \\
& \geqslant \sum_{\ell=1}^{\alpha^{-1}+1}\left(\begin{array}{l}
n \\
2
\end{array}\right)^{-1} \sum_{\{s, t\} \in\left(\begin{array}{c}
V \\
2
\end{array}\right)} \mathbb{1}_{[\operatorname{dist}(s, t) \geqslant \ell] .}
\end{aligned}
$$

For $\ell \in\left\{1, \ldots, \alpha^{-1}+1\right\}$, let $p_{\ell}=p_{\ell}\left(G_{n, d}\right)=\left(\begin{array}{c}n \\ 2\end{array}\right)^{-1} \sum_{\{s, t\} \in\left(\begin{array}{c}V \\ 2\end{array}\right)} \mathbb{1}_{[\operatorname{dist}(s, t) \geqslant \ell]}$. We evaluate $p_{\ell}$ using the following result.

Lemma 12. Consider $G_{n, d}$ of $d=(\beta+o(1)) n^{\alpha}$. Fix two constants $\alpha \in(0,1)$ and $\beta>0$ satisfying $\alpha^{-1} \in \mathbb{N}$. For any constant $k \in \mathbb{N}$, fix $2 k$ distinct vertices $s_{1}, \ldots, s_{k}, t_{1}, \ldots, t_{k}$. For any fixed $\ell_{1}, \ldots, \ell_{k} \in\left\{1, \ldots, \alpha^{-1}+1\right\}$, it holds that

$$
\lim _{n \rightarrow \infty} \operatorname{Pr}\left[\bigwedge_{i=1}^{k}\left[\operatorname{dist}\left(s_{i}, t_{i}\right) \geqslant \ell_{i}\right]\right]=\exp \left(-M \beta^{1 / \alpha}\right)
$$

where $M=\left|\left\{i \in\{1, \ldots, k\}: \ell_{i}=\alpha^{-1}+1\right\}\right|$.

We will prove Lemma 12 in Section 3.1. For $\ell \in\left\{1, \ldots, \alpha^{-1}+1\right\}$, let

$$
\mu_{\ell}= \begin{cases}1 & \text { if } 1 \leqslant \ell \leqslant \alpha^{-1} \\ \exp \left(-\beta^{1 / \alpha}\right) & \text { if } \ell=\alpha^{-1}+1\end{cases}
$$

From Lemma 12, we have

$$
\begin{aligned}
\mathbf{E}\left[p_{\ell}\right] & =\left(\begin{array}{l}
n \\
2
\end{array}\right)^{-1} \sum_{\{s, t\} \in\left(\begin{array}{c}
V \\
2
\end{array}\right)} \operatorname{Pr}[\operatorname{dist}(s, t) \geqslant \ell] \\
& =\operatorname{Pr}[\operatorname{dist}(1,2) \geqslant \ell]=\mu+o(1)
\end{aligned}
$$

and

$$
\begin{aligned}
& \mathbf{E}\left[p_{\ell}^{2}\right]=\left(\begin{array}{l}
n \\
2
\end{array}\right)^{-2} \sum_{\{s, t\},\left\{s^{\prime}, t^{\prime}\right\} \in\left(\begin{array}{c}
V \\
2
\end{array}\right)} \operatorname{Pr}\left[\operatorname{dist}(s, t) \geqslant \ell \wedge \operatorname{dist}\left(s^{\prime}, t^{\prime}\right) \geqslant \ell\right]
\end{aligned}
$$

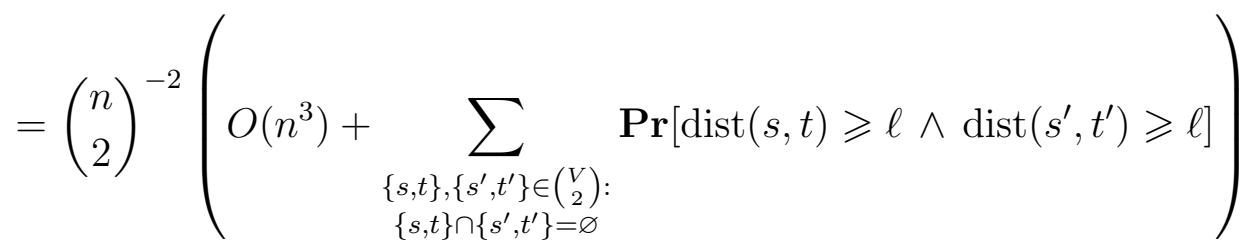

$$
\begin{aligned}
& =\operatorname{Pr}[\operatorname{dist}(1,2) \geqslant \ell \wedge \operatorname{dist}(3,4) \geqslant \ell]+o(1)=\mu^{2}+o(1) .
\end{aligned}
$$

From the Chebyshev inequality, for every constant $\epsilon>0$, we have

$$
\operatorname{Pr}\left[\left|p_{\ell}-\mathbf{E}\left[p_{\ell}\right]\right| \geqslant \epsilon\right] \leqslant \frac{\operatorname{Var}\left[p_{\ell}\right]}{\epsilon^{2}}=o(1) .
$$


Thus we obtain

$$
\operatorname{Pr}\left[\left|\left(\sum_{\ell=1}^{\alpha^{-1}+1} p_{\ell}\right)-\mu\right|>\epsilon\right] \leqslant \sum_{\ell=1}^{\alpha^{-1}+1} \operatorname{Pr}\left[\left|p_{\ell}-\mu_{\ell}\right|>\epsilon /\left(\alpha^{-1}+1\right)\right]=o(1) .
$$

Therefore, it holds w.h.p. that

$$
\operatorname{AD}\left(G_{n, d}\right) \geqslant \sum_{\ell=1}^{\alpha^{-1}+1} p_{\ell} \geqslant \mu-o(1)
$$

which completes the proof of Lemma 11.

\subsection{Distances of fixed vertex pairs of $G_{n, d}$}

This part is devoted to prove Lemma 12. We start with establishing the following result.

Lemma 13. Consider $G_{n, d}$ of $d=(\beta+o(1)) n^{\alpha}$ for constants $\alpha \in(0,1)$ and $\beta>0$. For two fixed distinct vertices $s$ and $t$, it holds w.h.p. that $\operatorname{dist}(s, t) \in\left\{\left\lceil\alpha^{-1}\right\rceil,\left\lfloor\alpha^{-1}\right\rfloor+1\right\}$.

Proof. For two fixed vertices $s, t$ of $G_{n, d}$ and an integer $\ell$, we denote by $\mathcal{P}$ the set of paths of length $\ell$ connecting $s$ and $t$ in a complete graph. Let $X_{\ell}=X_{\ell}\left(G_{n, d}\right)$ be the number of paths $P \in \mathcal{P}$ contained in $G_{n, d}$, that is,

$$
X_{\ell}=\left|\left\{P \in \mathcal{P}: P \subseteq G_{n, d}\right\}\right|
$$

Fix an integer $\ell$ satisfying $\ell \alpha<1$ (or equivalently, $\ell \leqslant\left\lceil\alpha^{-1}\right\rceil-1$ ). Then, from Lemma 8, we have

$$
\begin{aligned}
\mathrm{E}\left(X_{\ell}\right) & =\sum_{P \in \mathcal{P}} \operatorname{Pr}\left[P \subseteq G_{n, d}\right] \\
& =(1+o(1)) n^{\ell-1}\left(\frac{d}{n}\right)^{\ell} \\
& =o(1) .
\end{aligned}
$$

From the Markov's inequality, we obtain

$$
\begin{aligned}
\operatorname{Pr}[\operatorname{dist}(s, t) \leqslant \ell] & \leqslant \operatorname{Pr}\left[X_{1}+\cdots+X_{\ell}>0\right] \\
& \leqslant \sum_{i=1}^{\ell} \mathrm{E}\left(X_{i}\right) \\
& =o(1) .
\end{aligned}
$$

In other words, $\operatorname{dist}(s, t) \geqslant \ell+1 \geqslant\left\lceil\alpha^{-1}\right\rceil$ holds w.h.p.

On the other hand, from Equation $(9)$, we have $\operatorname{dist}(s, t) \leqslant \operatorname{diam}\left(G_{n, d}\right) \leqslant\left\lfloor\alpha^{-1}\right\rfloor+1$. This completes the proof of Lemma 13. 
Proof of Lemma 12. Fix an integer $k>0$ and $2 k$ distinct vertices $s_{1}, \ldots, s_{k}, t_{1}, \ldots, t_{k}$ of $G_{n, d}$, where $d=(\beta+o(1)) n^{\alpha}$. From Lemma 13, it holds w.h.p. that $\operatorname{dist}(s, t) \in$ $\left\{\alpha^{-1}, \alpha^{-1}+1\right\}$.

Suppose that $\ell_{1} \leqslant \alpha^{-1}$ and thus $\operatorname{dist}\left(s_{1}, t_{1}\right) \geqslant \ell_{1}$ holds w.h.p. Then we have

$$
\begin{aligned}
\operatorname{Pr}\left[\bigwedge_{i=2}^{k}\left[\operatorname{dist}\left(s_{i}, t_{i}\right) \geqslant \ell_{i}\right]\right]-\operatorname{Pr}\left[\operatorname{dist}\left(s_{1}, t_{1}\right)<\ell_{1}\right] & \leqslant \operatorname{Pr}\left[\bigwedge_{i=1}^{k}\left[\operatorname{dist}\left(s_{i}, t_{i}\right) \geqslant \ell_{i}\right]\right] \\
& \leqslant \operatorname{Pr}\left[\bigwedge_{i=2}^{k}\left[\operatorname{dist}\left(s_{i}, t_{i}\right) \geqslant \ell_{i}\right]\right]
\end{aligned}
$$

and thus

$$
\operatorname{Pr}\left[\bigwedge_{i=1}^{k}\left[\operatorname{dist}\left(s_{i}, t_{i}\right) \geqslant \ell_{i}\right]\right]=\operatorname{Pr}\left[\bigwedge_{i=2}^{k}\left[\operatorname{dist}\left(s_{i}, t_{i}\right) \geqslant \ell_{i}\right]\right]-o(1)
$$

Hence, we may assume that $\ell_{i}=\alpha^{-1}+1$ for all $i=1, \ldots, k$ (i.e., $M=k$ in Lemma 12).

Let $\mathcal{P}^{(i)}$ denote the set of $s_{i} t_{i}$-paths of length $\alpha^{-1}$ contained in the complete graph $K_{n}$. Define $X^{(i)}$ as the number of paths of $\mathcal{P}^{(i)}$ contained in $G_{n, d}$, that is,

$$
X^{(i)}=\mid\left\{P \in \mathcal{P}^{(i)}: P \subseteq G(n, p) \mid .\right.
$$

Then, we have

$$
\begin{aligned}
\operatorname{Pr}\left[\bigwedge_{i=1}^{k}\left[\operatorname{dist}\left(s_{i}, t_{i}\right) \geqslant \alpha^{-1}+1\right]\right] & =\operatorname{Pr}\left[\bigwedge_{i=1}^{k}\left[\operatorname{dist}\left(s_{i}, t_{i}\right) \geqslant \alpha^{-1}\right] \wedge \bigwedge_{i=1}^{k}\left[X^{(i)}=0\right]\right] \\
& =\operatorname{Pr}\left[\bigwedge_{i=1}^{k}\left[X^{(i)}=0\right]\right]-o(1) .
\end{aligned}
$$

We evaluate Equation (11) using the following result, which will be shown in Section 3.2

Lemma 14. Consider $G_{n, d}$ of $d=(\beta+o(1)) n^{\alpha}$, where $\alpha \in(0,1)$ and $\beta>0$ are any constants satisfying $\alpha^{-1} \in \mathbb{N}$. Fix $2 k$ distinct vertices $s_{1}, \ldots, s_{k}, t_{1}, \ldots, t_{k}$, where $k$ is any constant. For $i=1, \ldots, k$, let $X^{(i)}$ denote the number of $s_{i} t_{i}$-paths of length $\alpha^{-1} \in \mathbb{N}$ contained in $G(n, p)$. Fix arbitrary nonnegative integers $r_{1}, \ldots, r_{k}$. Then, it holds that

$$
\mathbf{E}\left[\prod_{i=1}^{k}\left(X^{(i)}\right)_{r_{i}}\right]=\left(\beta^{1 / \alpha}\right)^{R}+o(1)
$$

where $R=r_{1}+\cdots+r_{k}$.

From Lemma 14 and the Poisson approximation theorem (Lemma 7), we have

$$
\operatorname{Pr}\left[\bigwedge_{i=1}^{k}\left[X^{(i)}=0\right]\right]=\exp \left(-k \beta^{1 / \alpha}\right)+o(1)
$$


By combining Equations (11) and (12), we have

$$
\operatorname{Pr}\left[\bigwedge_{i=1}^{k}\left[\operatorname{dist}\left(s_{i}, t_{i}\right) \geqslant \alpha^{-1}+1\right]\right]=\exp \left(-k \beta^{1 / \alpha}\right)-o(1) .
$$

This completes the proof of Lemma 12 and thus Lemma 11.

\subsection{Proof of Lemma 14}

We first prove the following result and then show Lemma 14.

Lemma 15. Fix an integer $\ell \geqslant 1$ and consider $G(n, p)$ satisfying $(n p)^{\ell}=\Omega(n)$. Fix $2 k$ distinct vertices $s_{1}, \ldots, s_{k}, t_{1}, \ldots, t_{k}$, where $k$ is arbitrary constant. For $i=1, \ldots, k$, let $X^{(i)}$ denote the number of $s_{i} t_{i}$-paths of length $\ell \in \mathbb{N}$ contained in $G(n, p)$.

Then, for any fixed nonnegative integers $r_{1}, \ldots, r_{k}$,

$$
\mathbf{E}\left[\prod_{i=1}^{k}\left(X^{(i)}\right)_{r_{i}}\right]=n^{R(\ell-1)} p^{R \ell}\left(1 \pm O\left(\frac{1}{n p}\right)\right),
$$

where $R=r_{1}+\cdots+r_{k}$.

Corollary 16. Consider $G(n, p)$ of $p=(\beta+o(1)) n^{-1+\alpha}$, where $\alpha \in(0,1)$ and $\beta>0$ are any constants satisfying $\alpha^{-1} \in \mathbb{N}$. Fix arbitrary nonnegative integers $r_{1}, \ldots, r_{k}$. Then, it holds that

$$
\mathbf{E}\left[\prod_{i=1}^{k}\left(X^{(i)}\right)_{r_{i}}\right]=\left(\beta^{1 / \alpha}\right)^{R}+o(1)
$$

where $R=r_{1}+\cdots+r_{k}$.

Proof of Lemma 15. For a positive constant $k$, fix $2 k$ distinct vertices $s_{1}, \ldots, s_{k}, t_{1}, \ldots, t_{k}$. For every $i \in\{1, \ldots, k\}$, let $\mathcal{P}^{(i)}$ denote the set of all $s_{i} t_{i}$-paths of length $\ell$ contained in the complete graph. We denote by $X^{(i)}$ the number of paths of $\mathcal{P}^{(i)}$ contained in $G(n, p)$.

Fix nonnegative integers $k, r_{1}, \ldots, r_{k}$. We may assume that $r_{i}>0$ for every $i=$ $1, \ldots, k$. Let $\mathcal{A}=\left(\mathcal{P}^{(1)}\right)_{r_{1}} \times \cdots \times\left(\mathcal{P}^{(k)}\right)_{r_{k}}$. Each element $A \in \mathcal{A}$ is a tuple

$$
A=\left(\left(P_{1}^{(1)}, \ldots, P_{r_{1}}^{(1)}\right), \ldots,\left(P_{1}^{(k)}, \ldots, P_{r_{k}}^{(k)}\right)\right),
$$

where each $P_{j}^{(i)} \in \mathcal{P}_{i}$ is an $s_{i} t_{i}$-path of length $\ell$ and $P_{j}^{(i)} \neq P_{j^{\prime}}^{(i)}$ holds for every $i$ and $j \neq j^{\prime}$. For notational convention, we write $A=\left(P_{1}, \ldots, P_{R}\right) \in \mathcal{A}$. Since $r_{k}>0$, it holds that $P_{R} \in \mathcal{P}^{(k)}$.

For a tuple $A=\left(P_{1}, \ldots, P_{t}\right)$ of $t$ paths, let $E(A)=\bigcup_{i=1}^{t} E\left(P_{i}\right)$ and $V(A)=\bigcup_{i=1}^{t} V\left(P_{i}\right)$ (we will use induction on $R$ and hence we assume $t \leqslant R$ here). For $\mathcal{S} \subseteq \mathcal{A}$, we consider

$$
\Gamma_{\mathcal{S}}=\sum_{A \in \mathcal{S}} p^{|E(A)|}
$$


Note that $\mathbf{E}\left[\prod_{i=1}^{k}\left(X^{(i)}\right)_{r_{i}}\right]=\sum_{A \in \mathcal{A}} \operatorname{Pr}[E(A) \subseteq E(G(n, p))]=\Gamma_{\mathcal{A}}$. We claim

$$
n^{R(\ell-1)} p^{R \ell}\left(1-O\left(\frac{1}{n}\right)\right) \leqslant \Gamma_{\mathcal{A}} \leqslant n^{R(\ell-1)} p^{R \ell}\left(1+O\left(\frac{1}{n p}\right)\right),
$$

which completes the proof of Lemma 15.

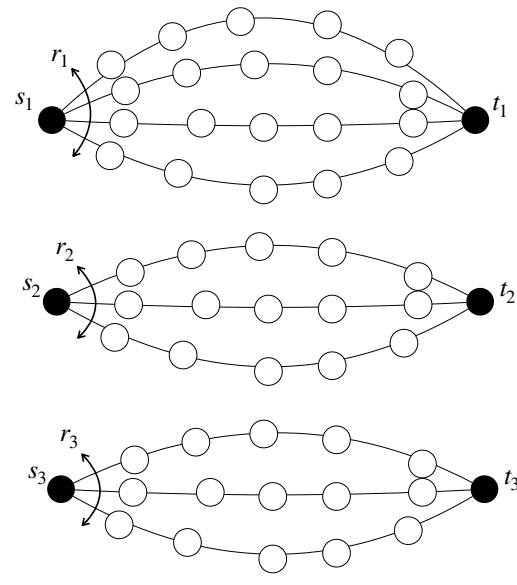

Figure 1: A tuple $A \in \mathcal{A} \backslash \mathcal{F}$.

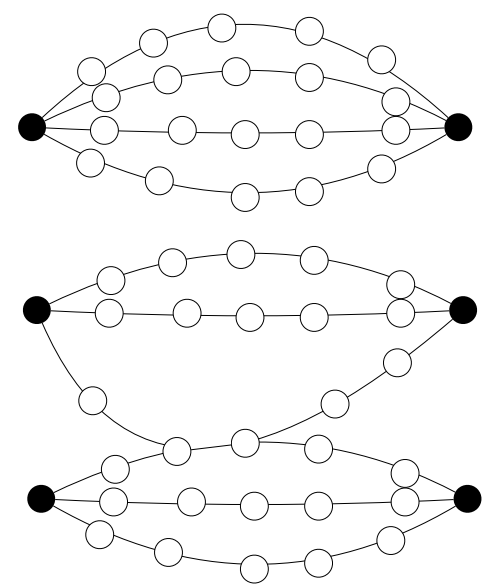

Figure 2: A tuple $A \in \mathcal{F}$.

For any $A \in \mathcal{A}$, it holds that $|E(A)| \leqslant R \ell$ and the equality holds if and only if any two distinct paths $P_{i}, P_{j}$ of $A$ shares no edges (see Figure 1). Let

$$
\begin{aligned}
\mathcal{F} & =\{A \in \mathcal{A}:|E(A)|<R \ell\} \\
& =\left\{\left(P_{1}, \ldots, P_{R}\right) \in \mathcal{A}: \exists i \neq j, E\left(P_{i}\right) \cap E\left(P_{j}\right) \neq \varnothing\right\} .
\end{aligned}
$$

Figure 2 illustrates an example. Then, $\Gamma_{\mathcal{A}}$ can be decomposed into

$$
\Gamma_{\mathcal{A}}=\Gamma_{\mathcal{F}}+\Gamma_{\mathcal{A} \backslash \mathcal{F}}
$$

The second term $\Gamma_{\mathcal{A} \backslash \mathcal{F}}$ satisfies

$$
\begin{aligned}
\Gamma_{\mathcal{A} \backslash \mathcal{F}} & =p^{R \ell}|\{A \in \mathcal{A}:|E(A)|=R \ell\}| \\
& \geqslant p^{R \ell} \mid\{A \in \mathcal{A}:|E(A)|=R \ell \text { and }|V(A)|=R(\ell-1)+2 k\} \mid \\
& =(n-2 k)_{R(\ell-1)} p^{R \ell} \\
& \geqslant n^{R(\ell-1)} p^{R \ell}\left(1-O\left(\frac{1}{n}\right)\right) .
\end{aligned}
$$

This implies the lower bound $\Gamma_{\mathcal{A}} \geqslant \Gamma_{\mathcal{A} \backslash \mathcal{F}} \geqslant n^{R(\ell-1)} p^{R \ell}\left(1-O\left(\frac{1}{n}\right)\right)$.

Now it suffices to bound $\Gamma_{\mathcal{A}}$ from above. Observe that $\Gamma_{\mathcal{A} \backslash \mathcal{F}}$ satisfies

$$
\Gamma_{\mathcal{A} \backslash \mathcal{F}}=p^{R \ell}|\{A \in \mathcal{A}:|E(A)|=R \ell\}| \leqslant n^{R(\ell-1)} p^{R \ell} .
$$

We show that this term is dominating in $\Gamma_{\mathcal{A}}$. Lemma 15 immediately follows from Equations (15) and (16) and the following result: 
Lemma 17. Suppose that $(n p)^{\ell}=\Omega(n)$. Define $\mathcal{F}$ as Equation (14). It holds that

$$
\Gamma_{\mathcal{F}}=O\left(\frac{n^{R(\ell-1)} p^{R \ell}}{n p}\right) .
$$

Proof. We use induction on $R$. For the base case of $R=1$, we have $\mathcal{F}=\varnothing$ and thus

$$
\begin{aligned}
& \Gamma_{\mathcal{A}} \leqslant n^{\ell-1} p^{\ell}, \\
& \Gamma_{\mathcal{F}}=0 .
\end{aligned}
$$

Suppose that $R \geqslant 2$ and that Lemma 17 holds for $R-1$. Note that Lemma 15 also holds for $R-1$ since Lemma 17 implies Lemma 15. Let

$$
\mathcal{A}^{\prime}=\left(\mathcal{P}^{(1)}\right)_{r_{1}} \times \cdots \times\left(\mathcal{P}^{(k-1)}\right)_{r_{k}-1} .
$$

Then, each element $A=\left(P_{1}, \ldots, P_{R}\right) \in \mathcal{A}$ can be decomposed into $A^{\prime}=\left(P_{1}, \ldots, P_{R-1}\right) \in$ $\mathcal{A}^{\prime}$ and $P_{R} \in \mathcal{P}^{(k)}$. Note that the edge set $E\left(A^{\prime}\right)$ for $A^{\prime} \in \mathcal{A}^{\prime}$ are defined in the same way as $E(A)$ and it holds that $\left|E\left(A^{\prime}\right)\right| \leqslant(R-1) \ell$. Let

$$
\mathcal{F}^{\prime}=\left\{A^{\prime} \in \mathcal{A}^{\prime}:\left|E\left(A^{\prime}\right)\right|<(R-1) \ell\right\} .
$$

By the induction assumption on $\mathcal{F}^{\prime}$ and $\mathcal{A}^{\prime}$, we have

$$
\Gamma_{\mathcal{A}^{\prime}} \leqslant n^{(R-1)(\ell-1)} p^{(R-1) \ell}\left(1+\frac{C_{1}}{n p}\right), \quad \Gamma_{\mathcal{F}^{\prime}} \leqslant C_{2}\left(\frac{n^{(R-1)(\ell-1)} p^{(R-1) \ell}}{n p}\right)
$$

for some constants $C_{1}, C_{2}>0$. For $A=\left(P_{1}, \ldots, P_{R}\right) \in \mathcal{F}$, let $A^{\prime}=\left(P_{1}, \ldots, P_{R-1}\right) \in \mathcal{A}^{\prime}$. Since $A \in \mathcal{F}$, either

(i) $E\left(P_{R}\right) \cap E\left(P_{i}\right) \neq \varnothing$ for some $1 \leqslant i<R$, or

(ii) $E\left(P_{R}\right) \cap E\left(A^{\prime}\right)=\varnothing$ and $E\left(P_{i}\right) \cap E\left(P_{j}\right) \neq \varnothing$ for some $1 \leqslant i<j<R$ (thus $\left.A^{\prime} \in \mathcal{F}^{\prime}\right)$ holds. Therefore, we have

$$
\begin{aligned}
\Gamma_{\mathcal{F}} & =\sum_{A \in \mathcal{F}} p^{|E(A)|} \\
& \leqslant \sum_{A^{\prime} \in \mathcal{A}^{\prime}} \sum_{\substack{P_{R} \in \mathcal{P}^{(k)}: \\
E(A) \cap E\left(P_{R}\right) \neq \varnothing}} p^{\left|E\left(A^{\prime}\right) \cup E\left(P_{R}\right)\right|}+\sum_{A^{\prime} \in \mathcal{F}^{\prime}} \sum_{\substack{P_{R} \in \mathcal{P}^{(k)}: \\
E\left(P_{R}\right) \cap E\left(A^{\prime}\right)=\varnothing}} p^{\left|E\left(A^{\prime}\right) \cup E\left(P_{R}\right)\right|} .
\end{aligned}
$$

From the induction assumption, the second term satisfies

$$
\begin{aligned}
\sum_{A^{\prime} \in \mathcal{F}^{\prime}} \sum_{\substack{P_{R} \in \mathcal{P}^{(k)}: \\
E\left(P_{R}\right) \cap E\left(A^{\prime}\right)=\varnothing}} p^{\left|E\left(A^{\prime}\right) \cup E\left(P_{R}\right)\right|} & =\sum_{A^{\prime} \in \mathcal{F}^{\prime}} p^{\left|E\left(A^{\prime}\right)\right|} \sum_{\substack{P_{R} \in \mathcal{P}^{(k)} ; \\
E\left(P_{R}\right) \cap E\left(A^{\prime}\right)=\varnothing}} p^{\left|E\left(P_{R}\right)\right|} \\
& \leqslant \Gamma_{\mathcal{F}^{\prime}} \cdot n^{\ell-1} p^{\ell}
\end{aligned}
$$


The first term can be rewritten as

$$
\sum_{A^{\prime} \in \mathcal{A}^{\prime}} \sum_{\substack{P_{R} \in \mathcal{P}^{(k)}: \\ E\left(A^{\prime}\right) \cap E\left(P_{R}\right) \neq \varnothing}} p^{\left|E\left(A^{\prime}\right) \cup E\left(P_{R}\right)\right|}=\sum_{A^{\prime} \in \mathcal{A}^{\prime}} p^{\left|E\left(A^{\prime}\right)\right|} \sum_{\substack{P_{R} \in \mathcal{P}^{(k)}: \\ E(A) \cap E\left(P_{R}\right) \neq \varnothing}} p^{\left|E\left(P_{R}\right) \backslash E\left(A^{\prime}\right)\right|} .
$$

Fix $A^{\prime}=\left(P_{1}, \ldots, P_{R-1}\right) \in \mathcal{A}^{\prime}$. Let $S=\left\{s_{1}, \ldots, s_{k}, t_{1}, \ldots, t_{k}\right\}$ be the endpoints of the paths and let $V_{1}=S \cup V\left(P_{1}\right) \cup \cdots \cup V\left(P_{R-1}\right)$. To bound the number of $P_{R}$ satisfying the condition (ii), we consider two cases: $E\left(P_{R}\right) \nsubseteq E\left(A^{\prime}\right)$ and $E\left(P_{R}\right) \subseteq E\left(A^{\prime}\right)$.

Case I. $\boldsymbol{E}\left(\boldsymbol{P}_{\boldsymbol{R}}\right) \nsubseteq \boldsymbol{E}\left(\boldsymbol{A}^{\prime}\right)$. The edge set $E\left(P_{R}\right) \cap E\left(A^{\prime}\right)$ forms a forest. Since $E\left(P_{R}\right) \nsubseteq$ $E\left(A^{\prime}\right)$, this forest is not connected and thus we have $\left|V\left(P_{R}\right) \cap V_{1}\right|-\left|E\left(P_{R}\right) \cap E\left(A^{\prime}\right)\right| \geqslant 2$. This yields

$$
\begin{aligned}
\left|V\left(P_{R}\right) \backslash V_{1}\right| & =\left|V\left(P_{R}\right)\right|-\left|V\left(P_{R}\right) \cap V_{1}\right| \\
& \leqslant \ell-\left|E\left(P_{R}\right) \cap E\left(A^{\prime}\right)\right|-1 .
\end{aligned}
$$

Let $\left|E\left(P_{R}\right) \cap E\left(A^{\prime}\right)\right|=t<\ell$. Then, $P_{R}$ consists of two type of vertices: at most $\ell-t-1$ from $V \backslash V_{1}$ and the others from $V_{1}$. Therefore, there are at most $n^{\ell-t-1}\left|V_{1}\right|^{t} \leqslant C^{t} n^{\ell-t-1}$ candidates for the path $P_{R}$ satisfying $\left|E\left(P_{R}\right) \cap E\left(A^{\prime}\right)\right|=t<\ell$, where $C=(R-1)(\ell+1)$ (recall that two endpoints of $P_{R}$ are fixed and thus they are not taken into account).

Case II. $\boldsymbol{E}\left(\boldsymbol{P}_{\boldsymbol{R}}\right) \subseteq \boldsymbol{E}\left(\boldsymbol{A}^{\prime}\right)$. We claim $A^{\prime} \in \mathcal{F}^{\prime}$. If not, it holds that $E\left(P_{i}\right) \cap E\left(P_{j}\right)=\varnothing$ for any $i<j<R$. Hence, $E\left(P_{R}\right) \subseteq E\left(A^{\prime}\right)$ implies $P_{R}=P_{i}$ for some $i<R$. This contradicts to the definition of $\mathcal{A}\left(P_{i} \neq P_{j}\right.$ for any $\left.i<j \leqslant R\right)$. Moreover, the number of $P_{R} \in \mathcal{P}^{(k)}$ satisfying $E\left(P_{R}\right) \subseteq E\left(A^{\prime}\right)$ is at most $\left|V_{1}\right|^{\ell-1} \leqslant C^{R(\ell-1)}$. Therefore, we have

$$
\begin{aligned}
& \sum_{A^{\prime} \in \mathcal{A}^{\prime}} \sum_{\substack{P_{R} \in \mathcal{P}^{(k)}: \\
E\left(A^{\prime}\right) \cap E\left(P_{R}\right) \neq \varnothing}} p^{\left|E\left(A^{\prime}\right) \cup E\left(P_{R}\right)\right|} \\
& \leqslant \sum_{A^{\prime} \in \mathcal{A}^{\prime}} p^{\left|E\left(A^{\prime}\right)\right|}\left(\sum_{t=1}^{\ell-1} \sum_{\substack{P_{R} \in \mathcal{P}^{(k)}: \\
\left|E(A) \cap E\left(P_{R}\right)\right|=t}} p^{\left|E\left(P_{R}\right) \backslash E\left(A^{\prime}\right)\right|}\right)+\sum_{A^{\prime} \in \mathcal{F}^{\prime}} p^{\left|E\left(A^{\prime}\right)\right|} C^{R(\ell-1)} \\
& \leqslant \sum_{A^{\prime} \in \mathcal{A}^{\prime}} p^{\left|E\left(A^{\prime}\right)\right|} \cdot \sum_{t=1}^{\ell-1} C^{t} n^{\ell-t-1} p^{\ell-t}+C^{R(\ell-1)} \Gamma_{\mathcal{F}^{\prime}} \\
& \leqslant \Gamma_{\mathcal{A}^{\prime}} \frac{C n^{\ell-1} p^{\ell}}{n p}\left(1+\frac{1.01 C}{n p}\right)+C^{R(\ell-1)} \Gamma_{\mathcal{F}^{\prime}}
\end{aligned}
$$

From Equations (17) to (19) and the induction assumption, we have

$$
\begin{aligned}
\Gamma_{\mathcal{F}} & \leqslant \Gamma_{\mathcal{F}^{\prime}} \cdot n^{\ell-1} p^{\ell}+\Gamma_{\mathcal{A}^{\prime}} \cdot \frac{C n^{\ell-1} p^{\ell}}{n p}\left(1+\frac{1.01 C}{n p}\right)+C^{R(\ell-1)} \Gamma_{\mathcal{F}^{\prime}} \\
& \leqslant O\left(\frac{n^{R(\ell-1)} p^{R \ell}}{n p}\right) .
\end{aligned}
$$


This completes the proof of Lemma 17 and thus Lemma 15 (Here, we have used the assumption that $\left.(n p)^{\ell}=\Omega(n)\right)$.

Proof of Lemma 14. Let $d=(1+o(1)) n p=(\beta+o(1)) n^{\alpha}$. From Lemma 8, we have $\operatorname{Pr}[H \subseteq G(n, p)]=(1+o(1)) \operatorname{Pr}\left[H \subseteq G_{n, d}\right]$ for any fixed graph $H$. Let $R=r_{1}+\cdots+r_{k}$ and $\mathcal{A}=\left(\mathcal{P}^{(1)}\right)_{r_{1}} \times \cdots \times\left(\mathcal{P}^{(k)}\right)_{r_{k}}$. We write each element $A \in \mathcal{A}$ as a tuple $A=\left(P_{1}, \ldots, P_{R}\right)$ of $R$ paths. Then, from Corollary 16, we have

$$
\begin{aligned}
\underset{G_{n, d}}{\mathbf{E}}\left[\prod_{i=1}^{k}\left(X^{(i)}\right)_{r_{i}}\right] & =\sum_{\left(P_{1}, \ldots, P_{R}\right) \in \mathcal{A}} \operatorname{Pr}\left[E\left(P_{1} \cup \cdots P_{R}\right) \subseteq G_{n, d}\right] \\
& =(1+o(1)) \sum_{\left(P_{1}, \ldots, P_{R}\right)} \operatorname{Pr}\left[E\left(P_{1} \cup \cdots P_{R}\right) \subseteq G(n, p)\right] \\
& =(1+o(1)) \underset{G(n, p)}{\mathbf{E}}\left[\prod_{i=1}^{k}\left(X^{(i)}\right)_{r_{i}}\right] \\
& =(\beta+o(1))^{1 / \alpha} .
\end{aligned}
$$

\section{Concentration of $\operatorname{AD}(G(n, p))$}

We prove Theorem 3. We use $\mathrm{AD}=\mathrm{AD}(G(n, p))$ and $\operatorname{diam}=\operatorname{diam}(G(n, p))$ as random variables. Let $D=\lceil\mu\rceil=\left\lfloor\alpha^{-1}\right\rfloor+1$. From Corollary 5, we have

$$
\begin{aligned}
\operatorname{Pr}[|\mathrm{AD}-\mu|>\epsilon] & \leqslant \operatorname{Pr}[|\mathrm{AD}-\mu|>\epsilon \mid \text { diam }=D] \mathbf{P r}[\operatorname{diam}=D]+\operatorname{Pr}[\operatorname{diam} \neq D] \\
& \leqslant \operatorname{Pr}[|\mathrm{AD}-\mu|>\epsilon \mid \text { diam }=D]+o(1)
\end{aligned}
$$

for any $\epsilon=\epsilon(n)>0$. Therefore, we may put the condition that diam $=D$.

For $i=1, \ldots, D$, let

$$
N_{i}=\left|\left\{\{s, t\} \in\left(\begin{array}{l}
V \\
2
\end{array}\right): \operatorname{dist}(s, t)=i\right\}\right|
$$

We will prove the following result in Section 4.1:

Lemma 18. Let $C>0$ be a sufficiently large constant and $\epsilon=\epsilon(n):=\sqrt{\frac{\log n}{n p}}$. Then, $\left|N_{i}-M_{i}\right| \leqslant C \epsilon M_{i}$ holds w.h.p. for all $i=1, \ldots, D-1$, where

$$
M_{i}= \begin{cases}\frac{(n p)^{i}}{n}\left(\begin{array}{l}
n \\
2
\end{array}\right) & \text { if } i<\alpha^{-1}, \\
\left(1-\exp \left(-\beta^{1 / \alpha}\right)\right)\left(\begin{array}{l}
n \\
2
\end{array}\right) & \text { if } i=\alpha^{-1} \in \mathbb{N} .\end{cases}
$$


An upper bound of AD. Conditioned on diam $=D$, it immediately holds that $\mathrm{AD} \leqslant$ diam $\leqslant D$. Thus, if $\alpha^{-1} \notin \mathbb{N}$, we have

$$
\mathrm{AD} \leqslant D=\mu
$$

with probability $1-\exp \left(-n^{\Omega(n)}\right)$.

Now we focus on the case where $\alpha^{-1} \in \mathbb{N}$. Let $\epsilon=C \sqrt{\frac{\log n}{n p}}$ for sufficiently large constant $C>0$. Conditioned on diam $=D$, Lemma 18 implies

$$
\begin{aligned}
N_{D} & =\left(\begin{array}{l}
n \\
2
\end{array}\right)-N_{1}-\ldots-N_{D-1} \\
& \leqslant(1+O(\epsilon)) \exp \left(-\beta^{1 / \alpha}\right)\left(\begin{array}{l}
n \\
2
\end{array}\right) .
\end{aligned}
$$

Therefore, conditioned on diam $=D$, we have

$$
\begin{aligned}
\left(\begin{array}{l}
n \\
2
\end{array}\right) \cdot \mathrm{AD} & =\sum_{i=1}^{D} i N_{i} \\
& \leqslant D N_{D}+(D-1)\left(\left(\begin{array}{l}
n \\
2
\end{array}\right)-N_{D}\right) \\
& =N_{D}+(D-1)\left(\begin{array}{l}
n \\
2
\end{array}\right) \\
& \leqslant(1+O(\epsilon)) \mu\left(\begin{array}{l}
n \\
2
\end{array}\right) .
\end{aligned}
$$

In other words, $\mathrm{AD} \leqslant \mu+O(\epsilon)$ holds w.h.p.

A lower bound of AD. Conditioned on diam $=D$, we have $N_{1}+\cdots+N_{D}=\left(\begin{array}{l}n \\ 2\end{array}\right)$ and thus

$$
\begin{aligned}
\left(\begin{array}{l}
n \\
2
\end{array}\right) \cdot \mathrm{AD} & =\sum_{i=1}^{D} i N_{i} \\
& =N_{1}+2 N_{2}+\cdots+(D-1) N_{D-1}+D\left(\left(\begin{array}{l}
n \\
2
\end{array}\right)-N_{1}-\cdots-N_{D-1}\right) \\
& =D\left(\begin{array}{l}
n \\
2
\end{array}\right)-(D-1) N_{1}-(D-2) N_{2}-\cdots-N_{D-1} \\
& \geqslant(1-O(\epsilon)) \mu\left(\begin{array}{l}
n \\
2
\end{array}\right) .
\end{aligned}
$$

In the last inequality, we used Lemma 18. This completes the proof of Theorem 3. 


\subsection{Proof of Lemma 18}

The proof of Lemma 18 is a slight modification of the proof of Theorem 7.1 of [14].

Consider $G(n, p)$ of $p=(\beta+o(1)) n^{-1+\alpha}$. Let $D=\left\lfloor\alpha^{-1}\right\rfloor+1$. We consider the breadth first search process on $G(n, p)$ from a fixed vertex. Fix a vertex $v$. For $k \geqslant 0$, let

$$
N_{k}(v)=\{w \in V: \operatorname{dist}(v, w)=k\} .
$$

Note that $N_{0}(v)=\{v\}$. For sufficiently large constant $C>0$ and $\epsilon:=\sqrt{\frac{\log n}{n p}}$, let $\mathcal{F}_{k}$ be the event of $G(n, p)$ that

$$
|| N_{i}(v)\left|-\frac{2 M_{i}}{n}\right| \leqslant \frac{C \epsilon M_{i}}{n} \text { for all } i=1, \ldots, k,
$$

where $M_{i}$ is given in Lemma 18. Note that $\mathcal{F}_{0}$ must hold. The degree of $v$ is denoted by $\operatorname{deg}(v)$. We denote by $\operatorname{Bin}(m, q)$ the binomial distributed random variable with $m$ trials and success probability $q$. Note that, if we are given $N_{0}(v), \ldots, N_{k-1}(v)$, the random variable $\left|N_{k}(v)\right|$ is distributed as a binomial random variable, that is,

$$
\left|N_{k}(v)\right| \sim \operatorname{Bin}\left(n-\sum_{i=0}^{k-1}\left|N_{i}(v)\right|, 1-(1-p)^{\left|N_{k-1}(v)\right|}\right) .
$$

Consider $\mathbf{E}\left[\left|N_{k}(v)\right| \mid \mathcal{F}_{k-1}\right]$. For every $k=1, \ldots, D-1$, conditioned on $\mathcal{F}_{k-1}$, we have

$$
n \geqslant n-\sum_{i=0}^{k-1}\left|N_{i}(v)\right| \geqslant(1-O(\epsilon)) n
$$

Here, recall that $(n p)^{D-1}=O(n)$. Using the inequality $\mathrm{e}^{-\frac{x}{1-x}} \leqslant 1-x \leqslant \mathrm{e}^{-x}$ for every $x \in[0,1)$ (c.f., Lemma 21.1 of [14]), we obtain

$$
1-(1-p)^{\left|N_{k-1}(v)\right|}= \begin{cases}(1 \pm O(\epsilon)) p(n p)^{k-1} & \text { if } k=1, \ldots, D-2 \\ (1 \pm O(\epsilon)) \exp \left(-\beta^{1 / \alpha}\right) & \text { if } k=D-1 .\end{cases}
$$

Therefore, we have

$$
\begin{aligned}
\mathbf{E}\left[\left|N_{k}(v)\right| \mid \mathcal{F}_{k-1}\right] & = \begin{cases}(1 \pm O(\epsilon))(n p)^{k} & \text { if } k=1, \ldots, D-2, \\
(1 \pm O(\epsilon)) \exp \left(-\beta^{1 / \alpha}\right) n & \text { if } k=D-1\end{cases} \\
& =(1 \pm O(\epsilon)) \frac{2 M_{k}}{n}
\end{aligned}
$$

From the Chernoff bound (Lemma 6), we have

$$
\begin{aligned}
\operatorname{Pr}\left[\mathcal{F}_{k} \mid \mathcal{F}_{k-1}\right] & \geqslant 1-\exp \left(-\Theta\left(\epsilon^{2}(n p)^{k}\right)\right) \\
& \geqslant 1-O\left(n^{-2}\right)
\end{aligned}
$$

if the constant $C$ is sufficiently large (recall that $C$ is the constant in the definition of $\left.\mathcal{F}_{k}\right)$. Therefore, $\mathcal{F}_{D-1}$ holds with probability $1-O\left(n^{-2}\right)$ for sufficiently large $C$. Taking the union bound, it holds w.h.p. that $\left|N_{i}(v)\right|=(1 \pm O(\epsilon)) \frac{2 M_{i}}{n}$ for all $v$. Consequently, we have $N_{i}=\frac{1}{2} \sum_{v \in V}\left|N_{i}(v)\right|=(1 \pm O(\epsilon)) M_{i}$, which completes the proof of Lemma 18 . 


\section{Acknowledgements}

The author would like to thank the anonymous referees for their careful work and useful comments.

\section{References}

[1] E. A. Bender, E. R. Canfield. The asymptotic number of non-negative integer matrices with given row and column sums. Journal of Combinatorial Theory, Series A 24(3), 296-301, 1978.

[2] V. D. Blondel, J.-L. Guillaume, J. M. Hendrickx, and R. M. Jungers. Distance distribution in random graphs and application to network exploration. Physical Review E, 76(066101), 2007.

[3] B. Bollobás. A probabilistic proof of an asymptotic formula for the number of labelled regular graphs. European Journal of Combinatorics, 1(4):311-316, 1980.

[4] B. Bollobás. The diameter of random graphs. Transactions of the American Mathematical Society, 267(1):41-52, 1981.

[5] B. Bollobás. The evolution of sparse graphs. Graph Theory and Combinatorics, Academic Press, 35-57, 1984.

[6] B. Bollobás. Random Graphs. Cambridge University Press, 2nd edition, 2001.

[7] B. Bollobás and W. F. de la Vega. The diameter of random regular graphs. Combinatorica, 2(2):125-134, 1982.

[8] F. Chung and L. Lu. The diameter of sparse random graphs. Advances in Applied Mathematics, 26(4):257-279, 2001.

[9] F. Chung and L. Lu. The average distance in a random graph with given expected degrees. Internet Mathematics, 1(1):91-113, 2004.

[10] B. Doerr. Probabilistic Tools for the Analysis of Randomized Optimization Heuristics. Theory of Evolutionary Computation-Recent Developments in Discrete Optimization, pages 1-87. Springer International Publishing, 2020.

[11] A. Dudek, A. Frieze, A. Ruciński, and M. Šileikis. Embedding the Erdős-Rényi hypergraph into the random regular hypergraph and Hamiltonicity. Journal of Combinatorial Theory, Series B, 122:719-740, 2017.

[12] P. Erdős, S. Fajtlowicz, and A. J. Hoffman. Maximum degree in graphs of diameter 2. Networks, 10(1):87-90, 1980.

[13] D. Fernholz and V. Ramachandran. The diameter of sparse random graphs. Random Structures and Algorithms, 31(4):482-516, 2007.

[14] A. Frieze and M. Karoński. Introduction to Random Graphs. Cambridge University Press, 2016.

[15] P. Gao, M. Isaev, and B. D. McKay. Sandwitching random regular graphs between binomial random graphs. Proceedings of the 31st Annual ACM-SIAM Symposium on Discrete Algorithms, pages 690-701, 2020. 
[16] P. Gao and N. C. Wormald. Uniform generation of random regular graphs. SIAM Journal on Computing, 46(4):1395-1427, 2017.

[17] A. J. Hoffman and R. R. Singleton. On Moore graphs with diameters 2 and 3. IBM Journal of Research and Development, 4(5):497-504, 1960.

[18] E. Katzav, M. Nitzan, D. ben Avraham, P. L. Krapisky, R. Künh, N. Ross, and O. Biham. Analytical results for the distribution of shortest path lengths in random networks. A Letters Journal Exploring the Frontiers of Physics, 111(26006), 2015.

[19] J. H. Kim, B. Sudakov, and V. H. Vu. Small subgraphs of random regular graphs. Discrete Mathematics, 307(15):1961-1967, 2007.

[20] J. H. Kim and V. H. Vu. Sandwiching random graphs: universality between random graph models. Advances in Mathematics, 188(2):444-469, 2004.

[21] J. H. Kim and V. H. Vu. Generating random regular graphs. Combinatorica, 26(6):683-708, 2006.

[22] V. Klee and D. Larman. Diameters of random graphs. Canadian Journal of Mathematics, 33:618-640, 1981.

[23] T. Łuczak. Random trees and random graphs. Random Structures and Algorithms, 13(3-4), 1998.

[24] B. D. McKay. Subgraphs of random graphs with specified degrees. Congressus Numerantium, 33, 213-223, 1990.

[25] B. D. McKay and N. C. Wormald. Uniform generation of random regular graphs of moderate degree. Journal of Algorithms, 11(1):52 - 67, 1990.

[26] M. Miller and J. Širáň. Moore graphs and beyond: A survey of the degree/diameter problem. The Electronic Journal of Combinatorics Dynamic Survey, (14), 2005.

[27] M. Nitzan, E. Katzav, R. Kühn, and O. Biham. Distance distribution in configuration-model networks. Physical Review E, 93(062309), 2016.

[28] O. Riordan and N. Wormald. The diameter of sparse random graphs. Combinatorics, Probability and Computing, 19:835-926, 2010.

[29] N. Shimizu. The diameter of dense random regular graphs. Proceedings of the 29th Annual ACM-SIAM Symposium on Discrete Algorithms, 1934-1944, 2018.

[30] A. Steger and N. C. Wormald. Generating random regular graphs quickly. Combinatorics, Probability and Computing, 8(4):377-396, 1999.

[31] R. van der Hofstad and G. Hooghiemstra. Universality for distances in power-law random graphs. Journal of Mathematical Physics, 49(125209), 2008.

[32] R. van der Hofstad, G. Hooghiemstra, and P. V. Mieghem. Distances in random graphs with finite variance degrees. Random Structures and Algorithms, 27(1):76$123,2005$.

[33] N. C. Wormald. Models of random regular graphs. Surveys in Combinatorics, 267:239-298, 1999. 